\title{
Quantum fluctuations in thin superconducting wires of finite length
}

\author{
H.P. Büchler, V.B. Geshkenbein, and G. Blatter \\ Theoretische Physik, ETH-Hönggerberg, CH-8093 Zürich, Switzerland
}

(Dated: October 31, 2018)

\begin{abstract}
In one dimensional wires, fluctuations destroy superconducting long-range order and stiffness at finite temperatures; in an infinite wire, quasi-long range order and stiffness survive at zero temperature if the wire's dimensionless admittance $\mu$ is large, $\mu>2$. We analyze the disappearance of this superconductor-insulator quantum phase transition in a finite wire and its resurrection due to the wire's coupling to its environment characterized through the dimensionless conductance $K$. Integrating over phase slips, we determine the flow of couplings and establish the $\mu-K$ phase diagram.
\end{abstract}

In systems with reduced dimensions (films and wires) fluctuations and disorder strongly influence on the superconducting transition temperature [1], eventually driving a superconductor-insulator (SI) transition which has attracted a lot of attention recently [2, 3, 4]. In 1D wires, the fluctuations of Cooper pairs appearing below the mean-field transition at $T_{c 0}$ define a finite resistance via nucleation of thermally activated phase slips [5, 6] and hence remove the finite temperature transition; superconductivity possibly survives only at zero temperature. The focus then is on the quantum nucleation of phase slips; their proliferation may trigger a zero temperature SI quantum phase transition [7, 8, 9, 10. While first attempts to observe quantum phase slips [7] are still debated due to the granular structure of the wires 11, 12, recent experiments on amorphous ultra-thin wires $[3,4]$ carry the signatures of a SI transition in a homogeneous system. In this letter, we analyze the bosonic Cooper pair fluctuations in realistic wires of finite length which are naturally coupled to their environment through their boundaries; we demonstrate how the SI transition is quenched in the finite system and reappears through its coupling to the environment.

Previous studies of bosonic fluctuations in infinite wires [9] have found a $T=0$ SI quantum phase transition at the critical value $\mu=2$ of the dimensionless admittance characterizing the wire's superconducting properties. At $T>0$ the system exhibits a finite resistance; ignoring an additional dissipative channel due to excited quasi-particles, we call the non-superconducting state an insulating one. On the other hand, real experiments are carried out on wires of finite length, $L \sim 0.5-2 \mu \mathrm{m}$ typically [3]. The wire's coupling to the environment through appropriate boundary conditions imposes a drastic change in the phase slip dynamics and modifies the wire's low-energy physics. A generic description is obtained by embedding the wire in a voltage driven loop with impedances $Z_{\mathrm{p}}(\omega)$ and $Z_{\mathrm{s}}(\omega)$ placed in parallel and in series, see Fig. 10 Here, we are mainly interested in thermodynamic aspects (involving the static resistances $R_{\mathrm{p}}$ and $R_{\mathrm{s}}$ ); furthermore we concentrate on the currentdriven limit with $R_{\mathrm{s}} \gg R_{\mathrm{p}}$, then $I=V / R_{\mathrm{s}}$ is fixed. The voltage driven case with $R_{\mathrm{p}} \ll R_{\mathrm{s}}$ and other mixed cases $\left(R_{\mathrm{s}}\right.$ of order $\left.R_{\mathrm{p}}\right)$ are easily derived from the current-
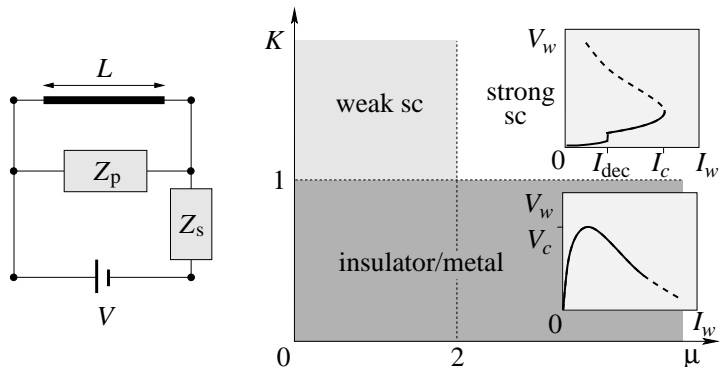

FIG. 1: Left: Setup with a quantum wire embedded in a voltage $(V)$ driven loop with parallel $\left(Z_{\mathrm{p}}\right)$ and serial $\left(Z_{\mathrm{s}}\right)$ impedances defining the environment. Right: Phase diagram with superconducting and insulating phases separated by a quantum phase transition at $K=1$. The superconducting phase splits into weak and strong regimes separated by a crossover at $\mu \approx 2$, the leftover of the SI transition in the infinite wire. The insets show sketches of the wire's $I_{\mathrm{w}}-V_{\mathrm{w}}$ characteristic at $T=0$. In the superconductor $(K>1)$ the algebraic characteristic is dominated by the environment at small currents $I<I_{\mathrm{dec}}$. A highly conducting shunt with $K \gg \mu$ allows to probe the wire above the deconfinement current $I_{\mathrm{dec}} ; I_{c}$ denotes the critical current of the wire. The insulator exhibits a Coulomb gap behavior below the critical voltage $V_{c}$.

driven solution via Kirchhoff's laws, see below.

The coupling to the environment changes the $T=0$ phase diagram of the infinite system, see Fig. 10 The SI transition at $\mu=2$ is turned into a crossover, while a new quantum phase transition appears at the critical value $K=R_{Q} / R_{\mathrm{p}}=1$ of the shunt's conductance ( $R_{Q}=\pi \hbar / 2 e^{2}$ denotes the quantum resistance). A well conducting shunt with $K>1$ relaxes the strain on the wire and produces a superconducting response with an algebraic $I-V$ characteristic, while a low conductance $K<1$ leads to the proliferation of phase slips and hence to an insulator. The characteristic response of the superconducting wire can be probed with a high conductance environment $K \gg \mu$ at large drive above the current $I_{\mathrm{dec}}$ where confined phase-slip pairs are separated.

In thin superconducting wires the plasma mode acquires a linear dispersion [13, 14] with sound velocity $c_{s}$ (this contrasts with the bulk case where the Coulomb interaction lifts the plasma mode to finite frequencies). 
The low frequency action describing the bosonic fluctuations of the Cooper pairs then takes the form [8, 9, 10]

$$
\frac{\mathcal{S}_{\mathrm{w}}}{\hbar}=\frac{\mu}{2 \pi} \int_{0}^{\hbar \beta} d \tau \int_{-L / 2}^{L / 2} d x\left[c_{s}\left(\partial_{x} \phi\right)^{2}+\frac{1}{c_{s}}\left(\partial_{\tau} \phi\right)^{2}\right]
$$

the first (second) term accounts for the kinetic (compression) energy (we assume a high energy cutoff $\hbar / \kappa$ limiting the validity of (10). Within a mean-field description the dimensionless admittance $\mu$ and the sound velocity $c_{s}$ are related to the $1 \mathrm{D}$ superfluid density $\rho_{s}=n_{s} S / 2 m^{*}$ and the Coulomb interaction between the Cooper pairs [9, 13], $\hbar \mu c_{s} / 2 \pi=\hbar^{2} \rho_{s} / 2$ and $\hbar \mu / 2 \pi c_{s}=(\hbar / 2 e)^{2} C / 2$ (here, $S$ is the wire's cross section and $C=\epsilon / \ln \left(d^{2} / S\right)$ is the capacitance per unit length, with $\epsilon$ the dielectric constant of the surrounding media placed a distance $d$ away). With $\lambda_{L}=\left(4 \pi n_{s} e^{2} / m c^{2}\right)^{1 / 2}$ the London penetration length we obtain $\mu \approx 30 \sqrt{C S} / \lambda_{L}$ and $c_{s}^{2} / c^{2} \approx 0.1 S / \lambda_{L}^{2} C$. Going beyond the mean-field level, both fermionic and bosonic high-energy fluctuations play a critical role in the proper determination of the low-energy action [15]; here, we assume such effects to be included in our choice of effective phenomenological parameters $\mu$ and $c_{s}$.

The coupling between the superconducting wire and the environment involves the boundary fields $\phi_{ \pm}(\tau) \equiv$ $\left.\phi_{ \pm}(x, \tau)\right|_{x=L / 2}$ where $\phi_{ \pm}(x, \tau)=\phi(x, \tau) \pm \phi(-x, \tau)$ : fluctuations in the phase difference $\phi_{-}$generate a voltage $V_{\mathrm{w}}$ across the wire inducing currents in the parallel shunts, while fluctuations in $\phi_{+}$account for charge accumulation. Here, we concentrate on the current-driven setup (see Fig. 1) described by the low-energy action

$$
\frac{\mathcal{S}_{\mathrm{e}}}{\hbar}=\frac{K}{2 \pi} \int \frac{d \omega}{4 \pi}|\omega|\left|\phi_{-}(\omega)\right|^{2}+\int_{0}^{\hbar \beta} d \tau \frac{I}{2 e} \phi_{-},
$$

with an ideal current source $\left(R_{\mathrm{s}} \gg R_{\mathrm{p}}\right)$ driving the system with the current $I$ and a parallel resistor with resistance $R_{\mathrm{p}}$ accounting for the dissipation $\left(K=R_{Q} / R_{\mathrm{p}}\right.$ is the dimensionless conductance of the shunt). Protecting the system from the measurement setup using appropriate filtering [16], we can neglect capacitive effects. The environment (2) does not account for an intrinsic, e.g., quasi-particle induced, dissipation in the superconducting wire; the latter contributes to the precise values of the parameters $\mu, c_{s}$, and $\lambda$ (the vortex fugacity, see below).

The statistical mechanics of the system 'wire plus environment' is determined by the partition function

$$
\mathcal{Z}=\int D[\phi] \exp [-\mathcal{S}(\phi) / \hbar]
$$

with $\mathcal{S}=\mathcal{S}_{\mathrm{w}}+\mathcal{S}_{\mathrm{e}}$. Its main contributions arise from the combination of Gaussian and topological fluctuations, so-called phase slips or instantons. Gaussian fluctuations destroy the superconducting long-range order as expressed by the logarithmically diverging phase correlator $\left\langle\left[\phi(x, \tau)-\phi\left(x, \tau^{\prime}\right)\right]^{2}\right\rangle \sim K^{-1} \ln \left|\tau-\tau^{\prime}\right|$; however, the surviving quasi-long range order is sufficient to allow for a finite phase stiffness, i.e., superconducting response. The latter is destroyed by the proliferation of phase slips, the process we are going to analyze in detail now.

Quantum phase slips are vortex-like solutions in $x, \tau$ space with finite winding $(\nu= \pm 1)$ around a core region of size $x_{c} \leq c_{s} \kappa$ and $\tau_{c} \leq \kappa$ where the superconducting gap $\Delta(x, \tau)$ drops to zero; outside this core region they satisfy the differential equation $\left[c_{s}^{2} \partial_{x}^{2}+\partial_{\tau}^{2}\right] \phi(\tau, x)=0$ within the wire and respect the boundary conditions

$$
K|\omega| \phi_{-}+\mu c_{s}\left[\partial_{x} \phi\right]_{-}=\pi I / e, \quad\left[\partial_{x} \phi\right]_{+}=0 .
$$

The first equation describes current conservation as given by Kirchhoff's law, with the supercurrent $I_{\mathrm{w}}=\left(e \mu c_{s} / \pi\right)$ $\left[\partial_{x} \phi\right]_{-}$through the wire and the dissipative current $V_{\mathrm{w}} / R_{\mathrm{p}}$ in the shunt adding up to the total external current $I$ (here, $\left.\left[\partial_{x} \phi\right]_{ \pm}(\tau) \equiv \partial_{x} \phi_{ \pm}(x, \tau)\right|_{x=L / 2}$ and $V_{\mathrm{w}}=$ $\hbar|\omega| \phi_{-} / 2 e$ is the voltage over the wire). The second equation accounts for charge neutrality; we ignore additional capacitive contributions here. Note that Kirchhoff's law maintains the form (4) when going over to the general situation with impedances both in series and in parallel, $I_{\mathrm{w}}+V_{\mathrm{w}} / R_{\mathrm{t}}=V / R_{\mathrm{s}}$ with $R_{\mathrm{t}}^{-1}=R_{\mathrm{p}}^{-1}+R_{\mathrm{s}}^{-1}$ the total conductance and the total current $I=V / R_{\mathrm{S}}$ is expressed through the driving voltage $V$. Hence once we know the solution $V_{\mathrm{w}}(I) \equiv R_{Q} \chi_{I}(I)$ to the current-driven problem (with $R_{\mathrm{p}}=R$ ), the solution $I_{\mathrm{w}}(V) \equiv \chi_{V}\left(V / R_{Q}\right)$ to the voltage-driven situation (with $R_{\mathrm{s}}=R$ ) follows from the relation $I-K \chi_{I}(I)=\chi_{V}(I / K)$, with $K=R_{Q} / R$.

The partition function (3) factorizes into Gaussian and topological parts, $\mathcal{Z}=\mathcal{Z}_{\mathrm{G}} \mathcal{Z}_{\text {top }}$, where the latter can be expanded in a series $\mathcal{Z}_{\text {top }}=\sum_{n=0}^{\infty}\left(\lambda^{n} / n !\right)^{2} \mathcal{Z}_{n}(G)$ with

$$
\mathcal{Z}_{n}=\int \frac{\prod_{m}^{2 n} d \tau_{m} d x_{m}}{\left(c_{s} \kappa^{2}\right)^{2 n}} \exp \left[\sum_{i \neq j} \nu_{i} G\left(x_{i}, \tau_{i}, x_{j}, \tau_{j}\right) \nu_{j}\right]
$$

describing a (neutral) gas of $n$ vortex-anti-vortex pairs, each contributing with a dimensionless action $G=$ $S_{\text {pair }} / \hbar$ (we assume $I=0$ ); in addition, each vortex-pair is weighted with the fugacity $\lambda^{2}$ accounting for the microscopic structure of the cores. Here, we assume that this factor is small, $\lambda \sim \exp \left(-A R_{Q} L / R_{N} \xi\right) \ll 1$ with $R_{N}$ the normal resistance of a wire, $\xi$ the superconducting coherence length, and $A$ a factor of order unity [9]

The interaction $G$ between vortex-anti-vortex pairs is the crucial quantity determining the system's behavior - here we only quote the relevant results, see Ref. 17] for details. For the infinite wire, the interaction between vortices $\phi_{k}= \pm \arg \left[\left(x-x_{k}\right)+i c_{s}\left(\tau-\tau_{k}\right)\right], k=1,2$, is logarithmic at all distances $\bar{x}=x_{1}-x_{2}, \bar{\tau}=\tau_{1}-\tau_{2}$,

$$
G(\bar{x}, \bar{\tau})=\mu \ln \left[\left(\bar{x}^{2} / c_{s}^{2}+\bar{\tau}^{2}\right) / \kappa^{2}\right] .
$$

A standard Kosterlitz-Thouless (KT) scaling analysis provides the RG equations [18] $\partial_{l} \mu=-4 \pi^{2} \mu^{2} \lambda^{2}$ and 
$\partial_{l} \lambda=(2-\mu) \lambda(l$ is the scaling parameter $)$ and the system undergoes a Berezinskii-Kosterlitz-Thouless transition 18 at $\mu=2$. For $\mu>2$, the number of free vortex-anti-vortex pairs is quenched and the wire is in the superconducting phase; for $\mu<2$ the vortex fugacity increases and free vortex-anti-vortex pairs proliferate the system turns insulating.

In a finite wire the solution has to respect the boundary condition (4). At low frequencies and vanishing drive these reduce to the Neumann boundary conditions, $\partial_{x} \phi_{\mathrm{N}}( \pm L / 2, \tau)=0$, i.e., the currents induced by phase slips cannot leave the wire. The solution $\phi_{\mathrm{N}}(x, \tau)$ derives from the $2 L$-periodic solution $\phi_{2 L-\mathrm{p}}$ using mirror vortices, $\phi_{\mathrm{N}}(x, \tau)=\phi_{2 L-\mathrm{p}}(x, \tau)+\phi_{2 L-\mathrm{p}}(L-x, \tau)$; in turn, the instanton solution with periodic boundary conditions $\phi_{2 L-\mathrm{p}}(x, \tau)=\phi_{2 L-\mathrm{p}}(x+2 L, \tau)$ derives from the solution in the infinite wire via conformal transformation. Fig. 2 illustrates the geometry of the phase slips; most remarkably, at large distances the mutual screening of the defect-pair is replaced by the screening via image charges. Hence the wire's contribution $G_{\mathrm{w}}$ to the action saturates at inter-vortex separations $\bar{\tau} \equiv\left|\tau_{2}-\tau_{1}\right|>L / \pi c_{s}$, resulting in asymptotically free vortices. Furthermore, their action can be minimized by moving them to the boundary such that $G_{\mathrm{w}}=0$.

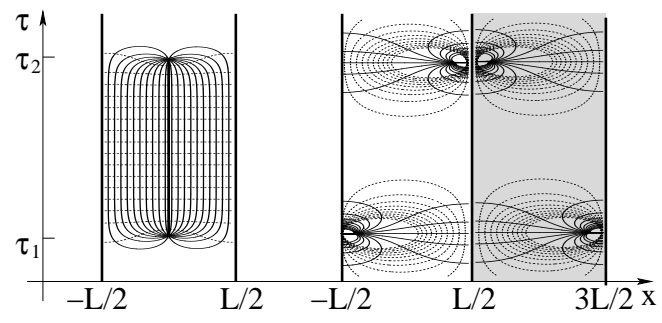

FIG. 2: Phase slip solutions with periodic (solid lines denote contours $\phi_{\mathrm{P}}=$ const., left) and Neumann ( $\phi_{\mathrm{N}}$, right) boundary conditions at intervortex distance $L / c_{s}<\bar{\tau}$. Left: for $K \gg \mu$, defects are screened mutually, the $2 \pi$ phase drop appears along the $x$-axis and drives a large current (dashed) through the highly conducting shunt; the resulting string confines defect pairs. Right: with $\mu \gg K$, defects are screened individually by their mirror images (grey underlaid), the $2 \pi$ phase drop appears along the $\tau$-axis and sets up displacement currents in the wire which cannot escape through shunt, hence charge accumulates at the boundary resulting in a large voltage over the shunt; the defects are asymptotically free.

In this situation the pair interaction is determined by the environment alone. Inserting the boundary field $\phi_{\mathrm{N}-}(\tau)$ describing two opposite kinks of shape $\pm 2 \arctan \left\{\sinh \left[\left(\pi c_{s} / L\right)\left(\tau-\tau_{k}\right)\right] / \cos \left(\pi x_{k} / L\right)\right\}, k=1,2$, into the action (2) we obtain the contribution

$$
G_{\mathrm{e}}\left(x_{1}, x_{2}, \bar{\tau}\right) \approx K \ln \left[\frac{\cos ^{2} \frac{\pi}{2 L}\left(x_{1}+x_{2}\right)+\left(\frac{\pi c_{s}}{2 L} \bar{\tau}\right)^{2}}{\cos \frac{\pi}{L} x_{1} \cos \frac{\pi}{L} x_{2}}\right]
$$

which diverges logarithmically at long time scales, but with a weight determined by the dimensionless conduc- tance $K$ of the parallel shunt. In the end, the largest contribution to the partition function arises from vortices nucleated at the boundary with an interaction $G \approx$ $G_{\mathrm{e}}=K \ln \left(\bar{\tau}^{2} / \kappa^{2}\right)$ and the problem maps to a system of charged particles in 1D with fugacity $\lambda$. The corresponding RG equations [19] take the form $\partial_{l} K=0$ and $\partial_{l} \lambda=(1-K) \lambda$; in contrast to the infinite wire, the prefactor $K$ of the logarithm is invariant under the RG flow and we obtain a transition at $K=1$ [20], with a superconducting phase for $K>1$, while for $K<1$ the vortices nucleated at the boundary drive the system insulating.

In the analysis above we have determined the shape of the vortex-anti-vortex pair assuming Neumann boundary conditions, i.e., no currents can leave the wire. Inserting this approximate solution back into (2) then has provided us with the pair-interaction $G \approx G_{\mathrm{e}}=2 K \ln (\bar{\tau} / \kappa)$. In order to check the consistency of this approximation we determine the correction $\delta \mathcal{S}$ due to the finite current $K|\omega| \phi_{-}$flowing through the shunt (cf. (44)),

$$
\frac{\delta \mathcal{S}}{\hbar}=-\frac{K}{4 \pi} \int \frac{d \omega}{2 \pi} \frac{|\omega|\left|\phi_{\mathrm{N}-}\right|^{2}}{1+(\mu / K)\left|\operatorname{coth}\left(\omega L / 2 c_{s}\right)\right|},
$$

with $\phi_{\mathrm{N}-} \approx(4 \pi / \omega) \sin (\omega \bar{\tau} / 2)$; performing the integral in (17) shows that the corrections are indeed small at low frequencies. For a setup with a poorly conducting shunt $(K \ll \mu)$ the corrections remain small at higher energies when $\bar{\tau}<L / \pi c_{s}, \delta S \sim-\mu(K / \mu)^{2} \ln (\bar{\tau} / \kappa)$.

However, in the opposite case $K \gg \mu$ where a highly conducting shunt protects the superconductor the corrections are large: in the intermediate regime $L / \pi c_{s}<\bar{\tau}<$ $\tau_{\mathrm{B}} \equiv K L / \pi \mu c_{s}$, we find $\delta S \sim-2 K \ln (\bar{\tau} / \kappa)+2 \pi \mu c_{s} \bar{\tau} / L$. The first term cancels the logarithmic interaction with the environment, while the second term describes linearly confined pairs. It is then appropriate to change strategy: for $K \gg \mu$ and high frequencies the boundary condition (41) reduces to $\phi_{-}=0$ and $\left[\partial_{x} \phi\right]_{+}=0$ for $I=0$ and the phase-field for the pair is given by the $L$-periodic solution $\phi_{L-\mathrm{p}}$, producing an interaction between the vortices

$$
G_{\mathrm{p}}(x, \tau)=\mu \ln \left[\left(\frac{L}{\pi c_{s} \kappa}\right)^{2}\left(\sinh ^{2} \frac{\pi c_{s} \bar{\tau}}{L}+\sin ^{2} \frac{\pi \bar{x}}{L}\right)\right]
$$

describing defect pairs linearly confined along the $\tau$-axis for distances $\bar{\tau}>L / \pi c_{s}$, cf. Fig. 2 At the same time, no voltage appears over the shunt and the contribution from the environment vanishes, thus $G \approx G_{\mathrm{p}}$. Hence, for a highly conducting shunt $K \gg \mu$ the interaction starts with a logarithm $G\left(\bar{\tau}<L / \pi c_{s}\right) \sim \mu \ln \left[\left(\bar{\tau}^{2}+\bar{x}^{2}\right) / \kappa^{2}\right]$, goes over into confinement $G\left(L / \pi c_{s}<\bar{\tau}<\tau_{\mathrm{B}}\right) \sim 2 \pi \mu c_{s} \bar{\tau} / L$, and ends with the low-frequency behavior $G\left(\tau_{\mathrm{B}}<\bar{\tau}\right) \sim$ $K \ln \left(\bar{\tau}^{2} / \kappa^{2}\right)$ determined by the environment.

In the end, we find that for all values of $K$ the lowenergy physics of the system is determined by the environment. On the other hand, the renormalization down to low energies depends on the ratio $\mu / K$ : for $K \ll \mu$ the interaction is entirely determined by the 
environment, while for $K \gg \mu$ the phase-slip pairs go through an intermediate regime of linear confinement inducing an exponential drop of the phase-slip fugacity, $\lambda\left(\tau_{\mathrm{B}}\right) \sim \lambda\left(L / \pi c_{s}\right) \exp \left[-G_{\mathrm{p}}\left(\tau_{\mathrm{B}}\right)\right]=\lambda\left(L / \pi c_{s}\right) \exp (-K) ;$ this drop in $\lambda$ manifests itself in the $I_{\mathrm{w}}-V_{\mathrm{w}}$-characteristic.

The above analysis results in a $T=0$ phase diagram as sketched in Fig. 10 The transition at $K=1$ separates a superfluid phase for $K>1$ from an insulating one at $K<1$. In addition, we distinguish between two different superconducting regimes at $K>1$ : for $\mu<2$ the fugacity for the nucleation of small $\left(\bar{\tau}<L / \pi c_{s}\right)$ vortexanti-vortex pairs is strongly increased as compared to the regime $\mu>2$, a leftover from the SI transition in the infinite wire which is relevant at temperatures $T>\hbar \pi c_{s} / L$ and drives $I>2 e \mu c_{s} / L$ probing these small scales.

With the interaction between defect pairs turning logarithmic on large scales $\bar{\tau}>\tau_{\mathrm{K}} \equiv \max \left(\kappa, \tau_{\mathrm{B}}\right)$, we find that the low-energy physics of the wire reduces to that of a Josephson junction with a parallel shunt $R$; the partition function is equivalent to that of a particle in a periodic potential with damping $\eta=K / 2 \pi[20$. The $I-V$ characteristic of such Josephson junctions has been studied in detail [20, 21]; below, we review the main results. The restricted validity of the effective action $\mathcal{S}=\mathcal{S}_{\mathrm{w}}+\mathcal{S}_{\mathrm{e}}$ to low energy scales limits this analysis to low temperatures $T<\hbar / \tau_{\mathrm{K}}$ and low currents $I<2 e K / \pi \tau_{\mathrm{K}}$.

Within the superconducting phase $K>1$, the $I-V$ characteristic at low drives $I$ is calculated perturbatively in the vortex fugacity $\lambda$ (with $\lambda\left(\tau_{\mathrm{K}}\right) \rightarrow \lambda$ the renormalized fugacity at $\left.\tau_{\mathrm{K}}\right)$. At finite temperatures the two energy scales $T$ and $\hbar \pi I / 2 e K$ define two regimes in the response, $V_{\mathrm{ps}}(\pi I / 2 e K \ll T / \hbar) \sim \lambda^{2} R_{Q} I\left[T \tau_{\mathrm{K}} / \hbar\right]^{2 K-2}$ and $V_{\mathrm{ps}}(T / \hbar \ll \pi I / 2 e K) \sim\left(\hbar \lambda^{2} / e \tau_{\mathrm{K}}\right)\left[I \tau_{\mathrm{K}} / e\right]^{2 K-1}$; the algebraic characteristic at $T=0$ turns into a linear response at finite $T$ and small drives $I$. We conclude, that in $1 \mathrm{D}$ wires superconductivity survives only at $T=0$ and under the condition of a good protection by a high conductance shunt with $K>1$. In addition, if $K \gg \mu$, the linear confinement in the regime $c_{s} / L<\bar{\tau}<\tau_{\mathrm{B}}$ produces a sharp voltage step $V_{\mathrm{dec}} \sim\left(\hbar \lambda^{2} / e \kappa\right)\left(L / c_{s} \kappa\right)\left[\pi \kappa c_{s} / L\right]^{2 \mu-2}$ at the deconfinement current $I_{\mathrm{dec}}=2 e \mu c_{s} / L$. At high drives $I>I_{\text {dec }}$ (and at temperatures $T>\hbar \pi c_{s} / L$ ) we start probing the wire (i.e., intervortex distances $\left.\bar{\tau}<\pi c_{s} / L\right)$ and the $I-V_{\mathrm{w}}$ characteristic is given by the result for the infinite system [9], $V_{\mathrm{ps}}\left(\pi c_{s} / L<\pi I / 2 \mu e \ll\right.$ $T / \hbar) \sim\left(\lambda^{2} R_{Q} I\right)\left(L / c_{s} \kappa\right)[T \kappa / \hbar]^{2 \mu-3}$ and $V_{\mathrm{ps}}\left(\pi c_{s} / L<\right.$ $T / \hbar \ll \pi I / 2 \mu e) \sim\left(\hbar \lambda^{2} / e \kappa\right)\left(L / c_{s} \kappa\right)[I \kappa / e \mu]^{2 \mu-2}$. The wire's $I_{\mathrm{w}}-V_{\mathrm{w}}$-characteristic derives from solution of the implicit equation $V_{\mathrm{w}}\left(I_{\mathrm{w}}\right)=V_{\mathrm{ps}}\left(I=I_{\mathrm{w}}+V_{\mathrm{w}} / R_{\mathrm{p}}\right)$.

In the insulating phase $K<1$ the phase slips describe tunneling of the phase difference $\phi_{-}$to neighboring states $\phi_{-} \pm 2 \pi$. In the limit $K \rightarrow 0$ this tunneling between periodic minima is coherent and leads to the formation of a Bloch band $\varepsilon(q)$ of width $W_{0}=\hbar \lambda / \kappa$, with $q$ the quasi-momentum associated to $\phi_{-}$. Applying a small current $I$, a voltage $2 e V_{\mathrm{w}}=\hbar\left\langle\dot{\phi}_{-}\right\rangle=\partial_{q} \varepsilon(q)$ is set up across the wire and all the current flows over the parallel shunt 21]; the system exhibits a linear response $V_{\mathrm{w}}=R I$. This behavior remains valid for finite $K<1$, but with a renormalized band width $16,21,22$ $W \approx W_{0}\left[W_{0} \tau_{\mathrm{K}} / \hbar\right]^{K /(1-K)}$, defining a linear response regime for small voltages $2 e V_{\mathrm{w}}<2 e V_{c}=\max \left(\partial_{q} \varepsilon\right) \sim W$. At higher drives, the voltage $V_{\mathrm{w}}$ decreases as the phase slips no longer block the wire and the current flows across both channels.

The SI quantum phase transition in the finite wire is different from the one in the infinite system; it is of the type inherent to finite systems coupled to a dissipative environment, e.g., the resistively shunted Josephson junction [20] or the dissipative two-state system [23]. The environment determines the wire's thermodynamic state and hence the overall shape of the $I_{\mathrm{w}}-V_{\mathrm{w}}$-characteristic, see Fig. 10 the wire's parameters $\mu$ and $\lambda$ make their appearance (through the voltage jump at $I_{\mathrm{dec}}$ and the wire-dominated characteristic at large currents $I>I_{\mathrm{dec}}$ ) only in the superconducting phase $K>1$ and for the case $K \gg \mu$ where a highly conducting shunt is protecting the wire. The insulating phase is established at small values $K<1$; phase-slips drive the current over the parallel shunt, resulting in an ohmic characteristic, until leakage through the wire sets in at $V_{c}$. Here, we have assumed a small vortex fugacity $\lambda$ and hence the observation (and consistent interpretation) of the characteristic voltage-peak requires a sensitive probe as $V_{c} \propto \lambda^{1 /(1-K)}$.

[1] A. Larkin, Ann. Phys. (Leipzig) 8, 785 (1999).

[2] D.B. Haviland et al., Phys. Rev. Lett. 62, 2180 (1989).

[3] A. Bezryadin et al., Nature 404, 971 (2000).

[4] C.N. Lau et al., Phys. Rev. Lett. 87, 217003 (2001).

[5] J.S. Langer and V. Ambegaokar, Phys. Rev. 164, 498 (1967).

[6] R. S. Newbower et al., Phys. Rev. B 5, 864 (1972).

[7] N. Giordano, Physica B 203, 460 (1994).

[8] V.A. Kashurnikov et al., Phys. Rev. B 53, 13091 (1996).

[9] A.D. Zaikin et al., Phys. Rev. Lett. 78, 1552 (1997); D.S. Golubev and A.D. Zaikin, Phys. Rev. B 64, 014504 (2001).

[10] F.W.J. Hekking and L.I. Glazman, Phys. Rev. B 55, 6551 (1997).

[11] P. Xiong et al., Phys. Rev. Lett. 78, 927 (1997).

[12] S.R. Renn, Phys. Rev. Lett. 76, 3400 (1996).

[13] J.E. Mooij and G. Schön, Phys. Rev. Lett. 55, 114 (1985).

[14] B. Camarota et al. Phys. Rev. Lett. 86, 480 (2001).

[15] Y. Oreg and A.M. Finkel'stein, Phys. Rev. Lett. 83, 191 (1999); Y. Oreg and E. Demler, cond-mat/0106645.

[16] J.S. Penttilä et al., Phys. Rev. Lett. 82, 1004 (1999); M. Watanabe and D. B. Haviland, Phys. Rev. Lett. 86, 5120 (2001).

[17] H.P. Büchler, Dissertation thesis, ETH Zürich (2003).

[18] J.M. Kosterlitz, J. Phys. C 7, 1046 (1974).

[19] S.A. Bulgadaev, Sov. Phys. JETP Lett. 39, 317 (1984).

[20] A. Schmid, Phys. Rev. Lett. 51, 1506 (1983). 
[21] G. Schön and A.D. Zaikin, Phys. Rep. 198, 237 (1990).

[22] G.L. Ingold and H. Grabert, Phys. Rev. Lett. 83, 3721

[23] A.J. Leggett et al., Rev. Mod. Phys. 59, 1 (1987). (1999). 